\title{
MARKET VALUE MARGIN VIA MEAN-VARIANCE HEDGING
}

\author{
BY \\ ANDREAS TSANAKAS, MARIO V. WÜTHRICH AND ALEŠ ČERNÝ
}

\begin{abstract}
We use mean-variance hedging in discrete time in order to value an insurance liability. The prediction of the insurance liability is decomposed into claims development results, that is, yearly deteriorations in its conditional expected values until the liability is finally settled. We assume the existence of a tradeable derivative with binary pay-off written on the claims development result and available in each development period. General valuation formulas are stated and, under additional assumptions, these valuation formulas simplify to resemble familiar regulatory cost-of-capital-based formulas. However, adoption of the meanvariance framework improves upon the regulatory approach by allowing for potential calibration to observed market prices, inclusion of other tradeable assets, and consistent extension to multiple periods. Furthermore, it is shown that the hedging strategy can also lead to increased capital efficiency.
\end{abstract}

\section{KEYWORDS}

Market value margin, mean-variance hedging, market consistent valuation, cost-of-capital, Solvency II.

\section{INTRODUCTION}

Market consistent valuation of insurance liabilities is a fundamental feature of new regulatory directives, as exemplified by the Swiss Solvency Test [23] and Solvency II, see [10]. Broadly speaking, regulatory valuation techniques distinguish between liabilities that can be replicated in deep, liquid and transparent markets and liabilities for which this is not possible. For the former type of liabilities, following standard financial arguments, the market values equal the initial costs of the replicating portfolios. For the latter, the market values are postulated as the sum of the expected present value of the liabilities and a market value margin that is set using cost-of-capital arguments. Thus, an explicit link is induced between capital assessment and valuation for regulatory purposes. 
The application of the above regulatory principle is not straightforward. First, the cost-of-capital rate used is a rather arbitrary, exogenously specified constant figure. Second, liabilities cannot be readily classified as perfectly replicable or completely non-replicable. It is usually the case that a liability can only be partly hedged and it is not entirely clear how the regulatory valuation approach should proceed in this case. A recent effort to reconcile cost-of-capital principles with replication arguments is given by Möhr [16], who obtains Solvency II valuation formulas as a special case. Third, given the long-term nature of many insurance liabilities, it is not clear what a multi-period extension of cost-of-capital valuation principles should be. Salzmann and Wüthrich [20] and Wüthrich and Merz [26] investigate alternative multi-period versions of cost-ofcapital valuation and show that conceptually consistent approaches can become computationally very expensive.

In this paper, valuation via mean-variance hedging of liabilities in discrete time is proposed; in particular, we use the solution in terms of sequential regression of Černý and Kallsen [3]. Mean-variance hedging identifies the selffinancing trading strategy that minimizes the quadratic deviation between the investment portfolio and the insurance liability at maturity. The general theory of mean-variance hedging is surveyed by Schweizer [21]. For more recent developments, we refer the reader to Črný and Kallsen [2] and references therein. Insurance applications of mean-variance hedging have been more common in life insurance where products demonstrate a higher dependence on instruments traded in financial markets, see Thomson [24], Dahl and Møller [5], and Delong [7]. Application to non-life insurance (see Delong and Gerrard [8]) is less frequent due to the greater difficulty in identifying suitable tradeable instruments.

However, the development of markets in insurance-linked securities, such as cat-bonds and weather derivatives, generates the possibility of at least partially hedging (non-life) insurance liabilities that are exposed to specific risks, such as those arising from natural catastrophes. We do not review the large literature on such securities and their markets here, but refer to Doherty [9] and Cummins [4]. Indicatively, we mention the progress that has been made in understanding the statistical behavior of observed cat-bond prices (Papachristou [19]) and attempt to derive reinsurance prices that are consistent with them (Haslip and Kaishev [12]).

Mean-variance hedging is related to other incomplete market pricing methods that have been applied to insurance. In particular, the discrete time riskminimization approach of Föllmer and Schweizer [11] determines locally optimal trading strategies. However, these are not necessarily self-financing as they allow the injection of capital at fixed times. For insurance applications of riskminimizing hedging strategies in continuous time, see Møller [17, 18]. An alternative approach has been to derive, via indifference arguments, market consistent versions of traditional actuarial premium calculations rules, such as the variance and standard deviation premium principles, see Schweizer [22].

We adopt a mean-variance hedging framework that considers a terminal liability (ultimate claim). Motivated by stochastic claims reserving methods in 
non-life insurance, the liability is decomposed into its expected value and claims development results representing yearly deteriorations (or improvements) in the liability's prediction using conditional expected values based on the latest information available (Merz and Wüthrich [14]). We then assume that the liability can be partially replicated by a tradeable instrument that pays 1 monetary unit in each period in the case that the claims development result exceeds a given threshold. Thus, investing in the derivative is a form of buying protection, loosely equivalent to issuing simple 1 -year bonds that are subordinated to the insurance liabilities. In addition, we allow for the possibility of investing in a risk-free asset and in a number of stocks. The analysis thus predicates on the assumption that a derivative as described above can be traded or indeed (its complement) issued by the liability holder. While buying the former will not always be a feasible option, issuing the latter is a possibility for several insurance operators. Furthermore, the valuation formulas can be seen as worst-case scenario valuation over the set of similar derivatives written on risks that are only partially correlated with the liability at hand (e.g. index-triggered cat-bonds).

General valuation formulas are derived for multiple time periods, multiple tradeable assets and general asset dynamics. Simplifying assumptions lead to more transparent and practical formulas: if (a) asset returns have stateindependent one-period Sharpe ratios, and (b) asset returns and claims development results are uncorrelated across different time periods, the market value of the liability is decomposed into valuations of the individual claims development results. Each of those can be written as a weighted sum of a Tail-Value-at-Risk (TVaR) measure applied to the claims development result and of capital asset pricing model (CAPM)-type terms corresponding to the other tradeable assets. If it is further assumed that only the derivative is tradeable, the valuation formula becomes very similar to multi-period cost-of-capital formulas found in Salzmann and Wüthrich [20].

While, in their simplest form, the valuation formulas obtained bear a strong similarity to the regulatory approach, their interpretation is different. Thus, we illustrate a potential set of alternative assumptions on which (regulatory) valuation may be based. The benefit of this approach is, firstly, to allow in a consistent manner the extension of liability valuation formulas to include multiple assets, partial replication, and multiple time periods. Secondly, by making explicit the assumptions needed to obtain simple and practically useful valuation formulas, it becomes transparent what the price paid for such simplification is.

In Section 2, the simple single-period and single-asset case is introduced and the corresponding valuation formulas are derived. We also show that the hedging approach used may lead to a more efficient use of capital, which is a positive side effect of the replication strategy. In Section 3, general results for the multiperiod and multi-asset case are presented and valuation formulas are derived for specific cases. Simple numerical examples illustrate the analysis. Finally, brief conclusions are given in Section 4.

Throughout the paper, we assume that the (conditional) second moments of all random variables considered exist. 


\section{SINGLE-PERIOD AND SINGLE-ASSET CASE}

\subsection{Preliminaries}

We start with a toy model to illustrate the key ideas of the paper. A single period is considered with two time points $t=0$ and $t=1$. There is an insurance liability $H \geq 0$ that has to be met at time $t=1$. At time $t=0$, the insurer of $H$ invests a total amount of $v$ monetary units in order to replicate $H$ as closely as possible. All assets and liabilities are considered in discounted units and there are two tradeable assets: a risk-free asset with price 1 at time $t=0$ and pay-off 1 at time $t=1$ and a risky asset with price $S_{0}$ at $t=0$ and pay-off $S_{1}$ at $t=1$. The risky asset's excess return is denoted by $X_{1}=S_{1} / S_{0}-1$.

Capital $\vartheta_{1}$ is invested in the risky asset $\left(\vartheta_{1} / S_{0}\right.$ units are bought) at time $t=0$ and the remainder of the initial wealth $v-\vartheta_{1}$ is invested in the risk-free asset. This asset portfolio generates value at time 1 given by $v+\vartheta_{1} X_{1}$. The optimal initial wealth $V_{0}$ and investment $\xi_{1}$ in the risky asset with respect to a quadratic loss function are calculated by minimizing the quadratic deviation between the liability and the asset portfolio's pay-off. That is,

$$
\left(V_{0}, \xi_{1}\right)=\arg \min _{\left(v, \vartheta_{1}\right)} \mathbb{E}\left(\left(v+\vartheta_{1} X_{1}-H\right)^{2}\right) .
$$

Since $V_{0}$ corresponds to the initial cost of replicating $H$ as closely as possible w.r.t. the quadratic loss, we will throughout this paper identify $V_{0}$ with the market consistent value of $H$ at time 0 .

Standard arguments yield the solution to optimization problem (1) which reads as

$$
\begin{aligned}
\xi_{1} & =\frac{\operatorname{Cov}\left(X_{1}, H\right)}{\operatorname{Var}\left(X_{1}\right)} \\
V_{0} & =\mathbb{E}(H)-\frac{\operatorname{Cov}\left(X_{1}, H\right)}{\operatorname{Var}\left(X_{1}\right)} \mathbb{E}\left(X_{1}\right) .
\end{aligned}
$$

In this simple setting, the value of $V_{0}$ reflects the CAPM price of $H$, where the risky asset with pay-off $S_{1}$ plays the role of the market portfolio.

In the sequel, the risk measures Value-at-Risk (VaR) and TVaR are used extensively. For a random variable $Z$ with continuous distribution function and a security level $\alpha \in(0,1)$, they are defined in the common way, see for instance McNeil et al. [13],

$$
\begin{aligned}
\operatorname{VaR}_{\alpha}(Z) & =\inf \{z \in \mathbb{R}: \mathbb{P}(Z \leq z) \geq \alpha\} \\
\operatorname{TVaR}_{\alpha}(Z) & =\frac{1}{1-\alpha} \int_{\alpha}^{1} \operatorname{VaR}_{\beta}(Z) d \beta=\mathbb{E}\left(Z \mid Z \geq \operatorname{VaR}_{\alpha}(Z)\right) .
\end{aligned}
$$

For the latter identity to hold true, we need to assume that $Z$ has a continuous distribution function, see Lemma 2.16 in McNeil et al. [13]. 


\subsection{Valuation formulas}

Now, a particular choice for the risky asset is made. For given random variable $Z_{1}$ and threshold $d_{1}$, we define

$$
S_{1}=\mathbf{1}_{D_{1}} \quad \text { with } \quad D_{1}=\left\{Z_{1} \geq d_{1}\right\} .
$$

Assume $p_{1}=\mathbb{E}\left(S_{1}\right)=\mathbb{P}\left(D_{1}\right) \in(0,1)$ and $S_{0}=q_{1} \in\left(p_{1}, 1\right)$ which implies $X_{1}=\mathbf{1}_{D_{1}} / q_{1}-1$.

There are two ways of considering such pay-offs in an insurance market. First, $S_{1}$ may be the pay-off from an index-linked insurance derivative (such as a weather derivative or an industry loss-triggered cat-bond) with $Z_{1}$ playing the role of the relevant index. The derivative considered will be such that $Z_{1}$ is a reasonable proxy for the liability $H$, hence we will require that $Z_{1}$ is positively correlated with $H$. In particular, the risky asset $S_{1}$ pays a positive return on the event $D_{1}$ associated with a large loss in $H$. Probability $p_{1}$ then is the realworld probability of such an event and $q_{1}$ is its risk-neutral probability implied by market prices. The condition $q_{1}>p_{1}$ is explained by $S_{1}$ playing the role of reinsurance for large losses. While $q_{1} \leq p_{1}$ would theoretically be possible, in practice the assumption generally holds that the cost of protection against an adverse event is higher than its expected value, due to risk aversion of the risk bearer.

Alternatively, consider the case that the holder of the liability $H$ sponsors a catastrophe bond, with $D_{1}$ being the triggering event. The bond structure is such that the holder of $H$ pays 1 monetary unit at time 1 if $D_{1}^{c}$ takes place and 0 units if $D_{1}$ occurs. Let $1-q_{1}$ be the price of the bond. Then, if the sponsor issues $\vartheta_{1} / q_{1}$ bonds, the gains from the trade are $-\frac{\vartheta_{1}}{q_{1}}\left(\mathbf{1}_{D_{1}^{c}}-\left(1-q_{1}\right)\right)=\vartheta_{1} X_{1}$. The solution to problem (1) now gives the optimal level of debt $\vartheta_{1}$ that should be issued. The bond will be constructed so as to maximize the correlation between the trigger $Z_{1}$ and the liability $H$. In particular, if an indemnity trigger is used, perfect positive correlation between $Z_{1}$ and $H$ can be achieved (see Papachristou [19]); otherwise, so-called basis risk remains.

For the tradeable asset (6), (3) and the identity $\operatorname{Var}\left(S_{1}\right)=p_{1}\left(1-p_{1}\right)$ yield

$$
V_{0}=\mathbb{E}(H)+\frac{q_{1}-p_{1}}{1-p_{1}}\left[\mathbb{E}\left(H \mid Z_{1} \geq d_{1}\right)-\mathbb{E}(H)\right] .
$$

Formula (7) can be further refined by choosing the indemnity-based trigger $Z_{1}=H-\mathbb{E}(H)$ (the threshold $d_{1}$ can now be interpreted as $d_{1}=\mathrm{VaR}_{1-p_{1}}(H)-$ $\mathbb{E}(H)$ ). Assuming that $H$ has a continuous distribution function, formula (7) becomes

$$
V_{0}=\mathbb{E}(H)+\frac{q_{1}-p_{1}}{1-p_{1}}\left[\operatorname{TVaR}_{1-p_{1}}(H)-\mathbb{E}(H)\right] .
$$

Therefore, valuation takes place according to a simple rule: "expected value plus a percentage of the excess of TVaR over the expected value". 
Even when the derivative is not indemnity-triggered (i.e. $H$ and $Z_{1}$ are not perfectly correlated), (8) can still be interpreted as a conservative upper bound on the value given in (7). To see this observe that the vectors $\left(H, \mathbf{1}_{\left\{H \geq \mathbb{E}(H)+d_{1}\right\}}\right)$ and $\left(H, \mathbf{1}_{\left\{Z_{1} \geq d_{1}\right\}}\right)$ have the same marginals, but the elements of the former are comonotonic. Hence $\mathbb{E}\left(H \mathbf{1}_{\left\{H \geq \mathbb{E}(H)+d_{1}\right\}}\right) \geq \mathbb{E}\left(H \mathbf{1}_{\left\{Z_{1} \geq d_{1}\right\}}\right)$ implies $\operatorname{TVaR}_{1-p_{1}}(H) \geq \mathbb{E}\left(H \mid Z_{1} \geq d_{1}\right)$, where the first inequality follows from Proposition 6.2.6 in Denuit et al. [6]. With this in mind, from now on we will always identify $Z_{1}$ with $H-\mathbb{E}(H)$.

Formula (8) bears a close resemblance to valuation formulas used under solvency regimes such as Solvency II and the Swiss Solvency Test, where the market consistent value of a liability is set equal to its expected value plus a risk loading deriving from a cost-of-capital charge, see European Commission [10] and Swiss Solvency Test [23]. If the regulator prescribes a translation invariant regulatory risk measure $\rho$ to support adverse events, the market consistent value under the cost-of-capital method equals

$$
V_{0}^{\mathrm{CoC}}=\mathbb{E}(H)+\lambda[\rho(H)-\mathbb{E}(H)],
$$

where $\lambda$ is the cost-of-capital rate. In Solvency II jargon, the quantity $\lambda[\rho(H)-\mathbb{E}(H)]$ is termed market value margin.

The valuation formulas (8) and (9) are structurally similar, both satisfying the rather pragmatic criterion of making an allowance for extreme tail events in the value of liability $H$ via a (tail) risk measure. However, they are derived using different economic arguments and thus are different in significant ways. In (9), $\lambda$ is an exogenously given constant, while $\frac{q_{1}-p_{1}}{1-p_{1}}$ in (8) is a potentially observable and market-sensitive quantity. In (9), $\rho(H)$ corresponds to the capital requirement for $H$, while the risk measure $\mathrm{TVaR}_{1-p_{1}}(H)$ is not associated with the assets held. In particular, for the optimal portfolio held, the investment in the risky asset would also be reflected in the regulatory capital requirement; this is a point to which we return in Section 2.3.

Example 1. The findings of Papachristou [19], who performed statistical analysis of catastrophe bond spreads at the time of issue, allow us to get a feeling for the potential range of the quantity $\frac{q_{1}-p_{1}}{1-p_{1}}$. In particular, the behavior of the "multiple" is studied, that is, the ratio of the spread to the annualized expected loss which in our simple model can be identified with the probability $p_{1}$. It is found that the multiple tends to decrease in $p_{1}$, reflecting a higher risk premium for protection against extreme events. Furthermore, it is shown how the multiple changes with time and responds to insurance events, e.g. a rise is observed after Hurricane Katrina in 2006. For the period of 2003-2008 studied and the sample of bonds considered, the multiple for bonds with $p_{1}=1 \%$ has tended to fluctuate between about four and eight. This implies that the spread $r_{B}=\frac{\mathbb{E}\left[\mathbf{1}_{1}^{c}\right]}{1-q_{1}}-1=\frac{1-p_{1}}{1-q_{1}}-1>0$ ranges from $4 \%$ to $8 \%$ and, consequently, $\frac{q_{1}-p_{1}}{1-p_{1}}$ varies from $3.8 \%$ to $7.4 \%$. Interestingly, the range contains the cost-of-capital rate $\lambda=6 \%$ favored by regulators, see TP.5.25 in [10]. 


\subsection{Hedging and capital efficiency}

The arguments presented above focused on deriving a risk-sensitive valuation formula as an alternative to what is proposed in current insurance regulation. However, we did not consider the change in portfolio risk after investment in the derivative with pay-off $S_{1}=\mathbf{1}_{D_{1}}$. This is an issue worth considering since the buyer of such a derivative would be interested in reducing the risk on the book and thus freeing up economic capital.

Let the solvency capital requirement be determined by a translation invariant risk measure $\rho$, such that $\rho(H-v)=\rho(H)-v$ for all $v \in \mathbb{R}$ and all random variables $H$ under consideration. Denote by $G_{1}$ the value of the optimal investment portfolio $\left(V_{0}, \xi_{1}\right)$ of (1) at time 1, i.e. $G_{1}=V_{0}+\xi_{1} X_{1}$. Then, trading in the derivative frees up capital as long as the cost $V_{0}$ of the trading strategy and the capital requirement for the hedged loss $H-G_{1}$ add up to less than the capital requirement for the unhedged loss $H$ :

$$
V_{0}+\rho\left(H-G_{1}\right) \leq \rho(H) \quad \Leftrightarrow \quad \rho\left(H-\left(G_{1}-V_{0}\right)\right) \leq \rho(H) .
$$

Noting that the portfolio that generates $G_{1}$ has initial price $V_{0}$, (10) states that investment in the portfolio can reduce the solvency capital requirement under the risk measure $\rho$, which looks similar to the indifference price for $G_{1}$ in an expected utility framework.

Nonetheless, it is by no means obvious that inequality (10) will generally be satisfied, since the trading strategy is formulated to replicate the liability $H$ as closely as possible in a quadratic norm and not specifically to minimize the capital requirement described by the risk measure $\rho$. Some situations where (10) holds are characterized in Proposition 1 stated below.

Proposition 1. Assume that $H$ has a continuous and strictly increasing distribution function on $\mathbb{R}_{+}$and let the risk measure $\rho$ be either $\mathrm{VaR}_{\alpha}$ or $\mathrm{TVaR}_{\alpha}$ at security level $\alpha \in(0,1)$. Define $k=\frac{1}{1-p_{1}} \mathrm{TVaR}_{1-p_{1}}(H-\mathbb{E}(H))$. We have $k>0$, and inequality (10) holds if and only if

$$
q_{1} k \leq \rho(H)-\rho\left(H-\mathbf{1}_{D_{1}} k\right) .
$$

In particular, the following hold:

(i) Assume that $\operatorname{VaR}_{1-p_{1}}(H)-k>0$. If $\alpha<1-p_{1}$ is small enough, such that $\operatorname{VaR}_{\alpha}(H) \leq \operatorname{VaR}_{1-p_{1}}(H)-k$, then:

- For $\rho \equiv \mathrm{VaR}_{\alpha}$, there is no $q_{1} \in(0,1)$ such that inequality (10) holds.

- For $\rho \equiv \mathrm{TVaR}_{\alpha}$, inequality (10) holds for all $q_{1} \in\left(p_{1}, \frac{p_{1}}{1-\alpha}\right)$.

(ii) If $\alpha>1-p_{1}$ is large enough, such that $\operatorname{VaR}_{\alpha}(H)-\operatorname{VaR}_{1-p_{1}}(H) \geq k$, then, for either of $\rho \equiv \mathrm{VaR}_{\alpha}$ and $\rho \equiv \mathrm{TVaR}_{\alpha}$, inequality (10) holds for all $q_{1} \in\left(p_{1}, 1\right)$ and the freed-up capital equals

$$
\rho(H)-V_{0}-\rho\left(H-G_{1}\right)=\left(1-q_{1}\right) k .
$$


Proof. Note that both VaR and TVaR are translation invariant and that $k>0$ by the properties of TVaR (e.g. see Property 2.4 .5 in Denuit et al. [6]). We have

$$
\begin{aligned}
G_{1}-V_{0}=\xi_{1} X_{1} & =\frac{\operatorname{Cov}\left(X_{1}, H\right)}{\operatorname{Var}\left(X_{1}\right)} X_{1}=\frac{\mathbf{1}_{D_{1}}-q_{1}}{1-p_{1}} \operatorname{TVaR}_{1-p_{1}}(H-\mathbb{E}(H)) \\
& =\left(\mathbf{1}_{D_{1}}-q_{1}\right) k .
\end{aligned}
$$

It follows that $\rho\left(H-\left(G_{1}-V_{0}\right)\right)=\rho(W)+q_{1} k$, where $W=H-\mathbf{1}_{D_{1}} k$. Thus, the freed-up capital can be written as $\rho(H)-\rho\left(H-\left(G_{1}-V_{0}\right)\right)=\rho(H)-\rho(W)-q_{1} k$, so that to satisfy inequality (10) we need requirement (11).

Let $d=d_{1}+\mathbb{E}(H)$, which implies $D_{1}=\{H \geq d\}$ and $\operatorname{VaR}_{1-p_{1}}(H)=d$. We have for $w \in \mathbb{R}$

$$
\begin{aligned}
\mathbb{P}(W \leq w) & =\mathbb{P}\left(W \leq w, D_{1}\right)+\mathbb{P}\left(W \leq w, D_{1}^{c}\right) \\
& =\mathbb{P}(d \leq H \leq w+k)+\mathbb{P}(H \leq \min \{w, d\}) .
\end{aligned}
$$

Continuity of the distribution function $F$ of $H$ and $k>0$ immediately imply

$$
\mathbb{P}(W \leq w)= \begin{cases}F(w), & w \leq d-k, \\ F(w+k)-F(d)+F(w), & d-k<w<d, \\ F(w+k), & d \leq w .\end{cases}
$$

It is easily seen that the distribution of $W$ is also continuous and strictly increasing. Moreover, when $\operatorname{VaR}_{\alpha}(H) \leq d-k$ (corresponding to Case i), it is $\operatorname{VaR}_{\alpha}(W)=\operatorname{VaR}_{\alpha}(H)$. On the other hand, when $\operatorname{VaR}_{\alpha}(H) \geq d+k$ (corresponding to Case ii), we have $\mathbb{P}\left(W \leq \operatorname{VaR}_{\alpha}(H)-k\right)=\mathbb{P}\left(H \leq \operatorname{VaR}_{\alpha}(H)\right)=\alpha$, such that $\operatorname{VaR}_{\alpha}(W)=\operatorname{VaR}_{\alpha}(H)-k$. We now deal with the two cases separately.

Case

(i) Assume $d-k>0$ and choose $\operatorname{VaR}_{\alpha}(H) \leq d-k$.

1. First, let $\rho \equiv \operatorname{VaR}_{\alpha}$. Then $\operatorname{VaR}_{\alpha}\left(H-\mathbf{1}_{D_{1}} k\right)=\operatorname{VaR}_{\alpha}(W)=\operatorname{VaR}_{\alpha}(H)$, such that condition (11) cannot be satisfied for any $q_{1}>0$ (note that $k>0$ ).

2. Now, let $\rho \equiv \mathrm{TVaR}_{\alpha}$. Observe that the vectors $\left(H, \mathbf{1}_{\left\{H \geq \mathrm{VaR}_{\alpha}(H)\right\}}\right)$ and $\left(H, \mathbf{1}_{\left\{W \geq \operatorname{VaR}_{\alpha}(W)\right\}}\right)$ have the same marginals, but the elements of the former are comonotonic. This implies inequality $\mathbb{E}\left(H \mathbf{1}_{\left\{H \geq \operatorname{VaR}_{\alpha}(H)\right\}}\right) \geq \mathbb{E}\left(H \mathbf{1}_{\left\{W \geq \operatorname{VaR}_{\alpha}(W)\right\}}\right)$, which follows from Proposition 6.2.6 in Denuit et al. [6]. Consider now, using $\operatorname{VaR}_{\alpha}(W)=\operatorname{VaR}_{\alpha}(H)$,

$$
\begin{aligned}
\operatorname{TVaR}_{\alpha}(H)-\operatorname{TVaR}_{\alpha}(W) & \left.=\frac{1}{1-\alpha}\left[\mathbb{E}\left(H \mathbf{1}_{\left\{H \geq \operatorname{VaR}_{\alpha}(H)\right\}}\right)\right)-\mathbb{E}\left(W \mathbf{1}_{\left\{W \geq \operatorname{VaR}_{\alpha}(W)\right\}}\right)\right] \\
& \geq \frac{1}{1-\alpha}\left[\mathbb{E}\left(H \mathbf{1}_{\left\{W \geq \operatorname{VaR}_{\alpha}(W)\right\}}\right)-\mathbb{E}\left(W \mathbf{1}_{\left\{W \geq \operatorname{VaR}_{\alpha}(W)\right\}}\right)\right] \\
& =\frac{k}{1-\alpha} \mathbb{E}\left(\mathbf{1}_{D_{1}} \mathbf{1}_{\left\{W \geq \operatorname{VaR}_{\alpha}(W)\right\}}\right)
\end{aligned}
$$




$$
\begin{aligned}
& =\frac{k}{1-\alpha} \mathbb{P}\left(H \geq \max \left\{d, k+\operatorname{VaR}_{\alpha}(H)\right\}\right) \\
& =\frac{k}{1-\alpha} \mathbb{P}(H \geq d)=\frac{p_{1}}{1-\alpha} k .
\end{aligned}
$$

Hence, by (11) it is sufficient to have $q_{1} \leq \frac{p_{1}}{1-\alpha}$ for inequality (10) to hold. Case (ii) Assume $\operatorname{VaR}_{\alpha}(H) \geq d+k$ which gives $\operatorname{VaR}_{\alpha}(W)=\operatorname{VaR}_{\alpha}(H)-k$.

1. First, let $\rho \equiv \operatorname{VaR}_{\alpha}$. The freed-up capital equals $\operatorname{VaR}_{\alpha}(H)-\operatorname{VaR}_{\alpha}(W)-q_{1} k=$ $k-q_{1} k$, which proves the stated result.

2. Now, consider $\rho \equiv \mathrm{TVaR}_{\alpha}$. For $\beta \in[\alpha, 1)$, monotonicity implies $\operatorname{VaR}_{\beta}(H) \geq$ $\operatorname{VaR}_{\alpha}(H) \geq d+k$. Therefore, $\operatorname{VaR}_{\beta}(W)=\operatorname{VaR}_{\beta}(H)-k$ for all $\beta \in[\alpha, 1)$ and by the integral identity for TVaR given in (5), the freed-up capital statement immediately follows from $\operatorname{VaR}_{\beta}(H)-\operatorname{VaR}_{\beta}(W)-q_{1} k=k-q_{1} k$.

Case (i) of Proposition 1 refers to the case where the security level $\alpha$ is so low that the risk reduction effected by the derivative is not reflected in the VaR measure, due to the risk measure's insensitivity to the extreme tail of the distribution of $H$. Thus, investing any amount in the derivative incurs a cost with no apparent benefit. When TVaR is used, the extreme tails are reflected in the risk measurement and the benefit from investing in the derivative is recognized, as long as the derivative is not too expensive ( $q_{1}$ is not too high). On the other hand, Case (ii) refers to the situation where the security level $\alpha$ is very high such that under all scenarios considered by the risk measure, the derivative produces a pay-off of 1 monetary unit, which is always higher than the price $q_{1}<1$. Consequently, a capital saving is always produced. However, the freed-up capital, as seen in (12), depends on the price $q_{1}$. Thus, if $q_{1}$ is close to its lowest level $p_{1}$, there is no market risk premium for the derivative and the freed-up capital is maximized. On the contrary, if $q_{1}$ is close to 1 , the market considers the event of the derivative paying as nearly certain, such that the derivative becomes very expensive, and investing in it produces only a small capital reduction.

Of course, in many cases it will be $-k<\operatorname{VaR}_{\alpha}(H)-\operatorname{VaR}_{1-p_{1}}(H)<k$, a case not fully characterized in Proposition 1 . The following numerical example shows that for realistic parameter choices, investment in the derivative will tend to be capital efficient.

Example 2. Let $H$ be log-normally distributed such that $\mathbb{E}(H)=$ $100, \operatorname{Var}(H)=20^{2}$. We consider two cases of the derivative, with $p_{1}=0.01$ and $p_{1}=0.05$. For illustrative purposes, we follow again Papachristou [19], choosing for $p_{1}=0.01$ (resp. $p_{1}=0.05$ ) a multiple of 6 (resp. 4), leading to $q_{1}=0.066$ (resp. $q_{1}=0.208$ ).

Table 1 summarizes the quantities needed for the valuation of the liability $H$ according to (8), as well as the value $k=\frac{1}{1-p_{1}} \mathrm{TVaR}_{1-p_{1}}(H-\mathbb{E}(H))$ appearing in Proposition 1. While for $p_{1}=0.05$, the risk measure $\mathrm{TVaR}_{1-p_{1}}(H)$ is substantially lower, this is compensated by a higher ratio $\frac{q_{1}-p_{1}}{1-p_{1}}$, such that the liability $H$ has a higher market value $V_{0}$ for $p_{1}=0.05$. 
TABLE 1

VALUATION OF A LOG-NORMAL LIABILITY WITH $\mathbb{E}(H)=100, \operatorname{Var}(H)=20^{2}$.

\begin{tabular}{lcc}
\hline$p_{1}$ & 0.01 & 0.05 \\
$q_{1}$ & 0.066 & 0.208 \\
$\mathrm{TVaR}_{1-p_{1}}(H)$ & 166.56 & 147.95 \\
$\frac{q_{1}-p_{1}}{1-p_{1}}$ & 0.057 & 0.167 \\
$V_{0}$ & 103.77 & 107.99 \\
$k$ & 67.23 & 50.47 \\
\hline
\end{tabular}

(a) $p_{1}=0.01$

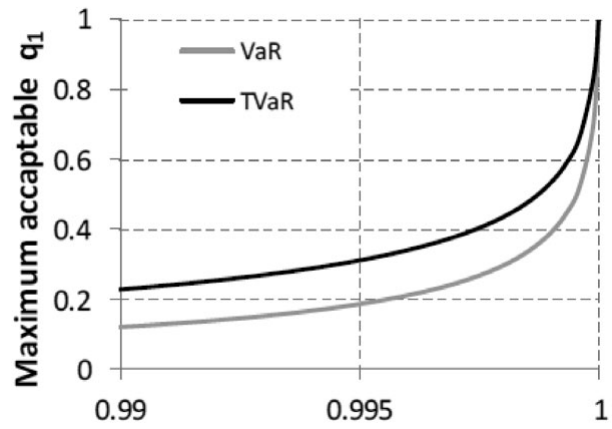

Confidence level $\alpha$ (b) $p_{1}=0.05$

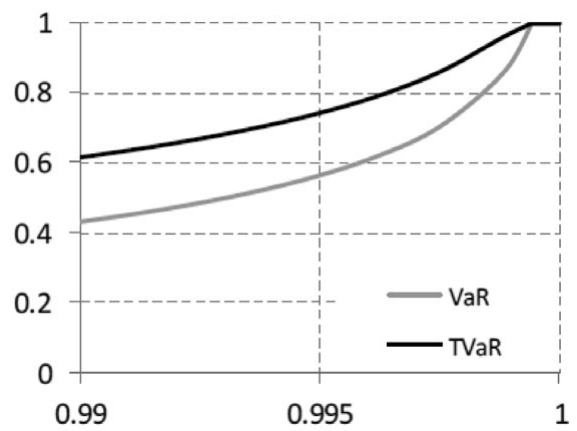

Confidence level $\alpha$

FIGURE 1: Maximum $q_{1}$ such that inequality (10) is satisfied.

It is easy to check that for all security levels $\alpha \in[0.99,0.999]$ (a plausible range for regulatory risk measurement), we have $\operatorname{VaR}_{\alpha}(H)-\operatorname{VaR}_{1-p_{1}}(H) \in$ $(0, k)$. From $(11)$, the maximum value of the price $q_{1}$ that leads to freeing up capital is given by the relation $q_{1} \leq\left(\rho(H)-\rho\left(H-\mathbf{1}_{D_{1}} k\right)\right) / k$, where $\rho \equiv \mathrm{VaR}_{\alpha}$ or $\rho \equiv \operatorname{TVaR}_{\alpha}$. The maximum such level of $q_{1}$ is plotted in Figure 1 against the security level $\alpha$ of the regulatory risk measure used for $\alpha \in[0.99,1)$. It is seen that in each case the value of $q_{1}$ is well below the plotted curves, such that for the plausible range of security levels $\alpha$, investment in the derivative indeed frees up capital.

\section{THE MULTI-PERIOD AND MULTI-ASSET CASE}

\subsection{Preliminaries}

We extend the previous setup to a model with several assets traded over multiple time periods. We consider a finite time horizon $T \in \mathbb{N}$ and a finite set of trading dates $\mathcal{T}=\{0,1, \ldots, T\}$. The filtered probability space is denoted by $(\Omega, \mathbb{P}, \mathcal{F}, \mathbb{F})$ with finite and discrete time filtration $\mathbb{F}=\left(\mathcal{F}_{t}\right)_{t \in \mathcal{T}}$ such that 
$\mathcal{F}_{0}=\{\emptyset, \Omega\}$ and $\mathcal{F}=\mathcal{F}_{T}$. The corresponding conditional expectations, variances, and covariances are denoted by $\mathbb{E}_{t}(X)=\mathbb{E}\left(X \mid \mathcal{F}_{t}\right), \operatorname{Var}_{t}(X)=\mathbb{E}_{t}\left(X^{2}\right)-$ $\mathbb{E}_{t}(X)^{2}, \operatorname{Cov}_{t}(X, Y)=\mathbb{E}_{t}(X Y)-\mathbb{E}_{t}(X) \mathbb{E}_{t}(Y)$ for $t \in \mathcal{T}$.

The insurance liability is represented by a non-negative, $\mathcal{F}_{T}$-measurable, square-integrable random variable $H \in L^{2}(\mathbb{P})$. We assume that we have $n \in \mathbb{N}$ tradeable risky assets with price processes represented by the $n$-dimensional, $\mathbb{F}$-adapted stochastic process $\left(S_{t}\right)_{t \in \mathcal{T}}$. Denote the elements of $S_{t}$ by $S_{t}^{(i)}, i=$ $1, \ldots, n$ and let $S_{t}^{(i)}>0 . X_{t}$ is then the vector of one-period excess returns with elements $X_{t}^{(i)}=S_{t}^{(i)} / S_{t-1}^{(i)}-1$. We assume that $\mathbb{E}_{t}\left(X_{t+1}^{(i)} X_{t+1}^{(j)}\right)<\infty$ for all $i, j$ and $t<T$, and that the returns of traded assets are linearly independent such that the matrices $\left\{\mathbb{E}_{t}\left(X_{t+1}^{(i)} X_{t+1}^{(j)}\right)\right\}_{1 \leq i, j \leq n}$ have full rank. For any vector $y \in \mathbb{R}^{n}$, $y^{\prime}$ denotes the transpose of $y$.

An $\mathcal{F}_{0}$-measurable initial endowment $v$ is given. A trading strategy $\vartheta=$ $\left(\vartheta_{t}\right)_{t \in \mathcal{T} \backslash\{0\}}$ is an $n$-dimensional and $\mathbb{F}$-previsible process. The value at time $t>0$ of an investment portfolio with initial endowment $v$ and trading strategy $\vartheta$ is

$$
G_{t}^{v, \vartheta}=v+\sum_{k=1}^{t} \vartheta_{k}^{\prime} X_{k} .
$$

By its construction, the portfolio (13) is self-financing. Only strategies such that $G_{T}^{v, \vartheta} \in L^{2}(\mathbb{P})$ are admitted; for a detailed technical discussion of admissibility, see Černý and Kallsen [3].

Directly extending the discussion in Section 2, the aim is to derive the optimal initial endowment and trading strategy such that the quadratic deviation between the final portfolio value $G_{T}^{v, \vartheta}$ and the liability $H$ is minimized. In other words, we need to solve optimization problem:

$$
\arg \min _{(v, \vartheta)} \mathbb{E}_{0}\left(\left(G_{T}^{v, \vartheta}-H\right)^{2}\right) .
$$

The solution to problem (14) is provided by Theorem 8.7 in Černý and Kallsen [3].

Theorem 2. The process given by the recursion $L_{T}=1$ and for $0<t \leq T$

$$
L_{t-1}=\mathbb{E}_{t-1}\left(L_{t}\right)-\mathbb{E}_{t-1}\left(L_{t} X_{t}^{\prime}\right)\left(\mathbb{E}_{t-1}\left(L_{t} X_{t} X_{t}^{\prime}\right)\right)^{-1} \mathbb{E}_{t-1}\left(L_{t} X_{t}\right)
$$

is $(0,1]$-valued and the probability measure $\mathbb{P}^{*}$, defined by

$$
\frac{d \mathbb{P}^{*}}{d \mathbb{P}}=\prod_{t=1}^{T} \frac{L_{t}}{\mathbb{E}_{t-1}\left(L_{t}\right)},
$$


is well defined. Let $\mathbb{E}_{t-1}^{*}(\cdot)$ denote conditional expectations under $\mathbb{P}^{*}$. The following processes for $0<t \leq T$ are well defined:

$$
\begin{aligned}
a_{t}^{*} & =\mathbb{E}_{t-1}^{*}\left(X_{t}^{\prime}\right)\left(\mathbb{E}_{t-1}^{*}\left(X_{t} X_{t}^{\prime}\right)\right)^{-1}, \\
b_{t}^{*} & =a_{t}^{*} \mathbb{E}_{t-1}^{*}\left(X_{t}\right), \\
V_{t-1}^{*} & =\mathbb{E}_{t-1}^{*}\left(\frac{1-a_{t}^{*} X_{t}}{1-b_{t}^{*}} V_{t}^{*}\right), \quad V_{T}^{*}=H, \\
\xi_{t}^{*} & =\mathbb{E}_{t-1}^{*}\left(\left(V_{t}^{*}-V_{t-1}^{*}\right) X_{t}\right)^{\prime}\left(\mathbb{E}_{t-1}^{*}\left(X_{t} X_{t}^{\prime}\right)\right)^{-1} .
\end{aligned}
$$

For initial endowment $v$ define the trading strategy $\phi(v)=\left(\phi_{t}(v)_{t}\right)_{t \in \mathcal{T} \backslash\{0\}}$ iteratively by

$$
\phi_{t}(v)=\xi_{t}^{*}+a_{t}^{*}\left(V_{t-1}^{*}-G_{t-1}^{v, \phi(v)}\right) .
$$

Then, the pair $\left(V_{0}^{*}, \phi\left(V_{0}^{*}\right)\right)$ solves the optimization problem (14).

The probability measure $\mathbb{P}^{*}$ is termed the opportunity-neutral measure. The opportunity-neutral measure $\mathbb{P}^{*}$ is not a martingale measure. Switching to $\mathbb{P}^{*}$ is necessary in the case that asset returns are not independent in order to compensate for one-period Sharpe ratios at a given time not being the same in all states (see Černý and Kallsen [3]). In the case $\mathbb{P}^{*}=\mathbb{P}$, we can keep the same notation as in Theorem 2 after dropping the superscripts * from all variables. The probability measure $\mathbb{P}^{*}$ reduces to $\mathbb{P}$ if and only if the product of $b_{t}=\mathbb{E}_{t-1}\left(X_{t}^{\prime}\right)\left(\mathbb{E}_{t-1}\left(X_{t} X_{t}^{\prime}\right)\right)^{-1} \mathbb{E}_{t-1}\left(X_{t}\right)$ over all $t$ is constant (see Cerný and Kallsen [2], Proposition 3.28). A sufficient condition for this is to require that the maximal one-period Sharpe ratio for each time step is known at time zero, equivalently $b_{t}$ is $\mathcal{F}_{0}$-measurable. Independence of asset returns is a substantially stronger condition; one can for example achieve constant Sharpe ratios in stochastic volatility models so that the returns are not i.i.d. but the $b_{t} \mathrm{~s}$ ' remain deterministic. Independence is a sufficient (but not necessary) condition for both $a_{t}$ and $b_{t}$ to be $\mathcal{F}_{0}$-measurable, that is, state independent. It also noted that the more general form of Theorem 2 is given in terms of price increments rather than returns; we use the current form (requiring $S_{t}>0$ ) for practical reasons, as the dynamics of asset returns, rather than prices, are typically specified.

\subsection{Valuation of an insurance liability}

We work toward deriving multi-period valuation formulas, generalizing those of Section 2. First, we decompose the $\mathcal{F}_{T}$-measurable liability $H \in L^{2}(\mathbb{P})$ as

$$
H=\mathbb{E}_{0}(H)+Y_{1}+\cdots+Y_{T} \quad \text { with } \quad Y_{t}=\mathbb{E}_{t}(H)-\mathbb{E}_{t-1}(H),
$$

where $Y_{t}$ is termed the claims development result, see Merz and Wüthrich [14]. The notion of the claims development result is based on the understanding that insurance companies need to close their books after every period. At time $t$, they 
will book the so-called best-estimate liability $\mathbb{E}_{t}(H)$, updating the previous prediction $\mathbb{E}_{t-1}(H)$. The resulting adjustment of the best estimate produces a claims development result of $Y_{t}$ in period $t$, which may be a gain or a loss. Essentially, $Y_{t}$ corresponds to the single-period risk exposure of the holder of $H$ and the regulator asks for a risk measure to support possible shortfalls in $Y_{t}$ in period $t$. Since the time series $Y_{1}, \ldots, Y_{T}$ is formed by the innovations of a martingale, its elements are uncorrelated and have zero mean. For the rest of the paper, we will assume that $Y_{t}$ has a continuous and strictly increasing distribution.

For such a decomposition of the liability $H$, direct application of Theorem 2 gives a general valuation formula.

Proposition 3. For $H$ as in (15), the optimal initial endowment of Theorem 2 becomes

$$
V_{0}^{*}=\mathbb{E}_{0}(H)+\sum_{t=1}^{T} \mathbb{E}_{0}^{*}\left(\prod_{i=1}^{t} \frac{1-a_{i}^{*} X_{i}}{1-b_{i}^{*}} Y_{t}\right) .
$$

Proof. From Theorem 2, we have (noting that $\mathbb{E}_{t-1}^{*}\left(\left(1-a_{t}^{*} X_{t}\right) /\left(1-b_{t}^{*}\right)\right)=$ 1 and $V_{T}^{*}=H$ ),

$$
\begin{aligned}
V_{T-1}^{*}= & \mathbb{E}_{T-1}^{*}\left(\frac{1-a_{T}^{*} X_{T}}{1-b_{T}^{*}} H\right)=\mathbb{E}_{0}(H)+\sum_{t=1}^{T-1} Y_{t}+\mathbb{E}_{T-1}^{*}\left(\frac{1-a_{T}^{*} X_{T}}{1-b_{T}^{*}} Y_{T}\right), \\
V_{T-2}^{*}= & \mathbb{E}_{T-2}^{*}\left(\frac{1-a_{T-1}^{*} X_{T-1}}{1-b_{T-1}^{*}} V_{T-1}\right) \\
= & \mathbb{E}_{0}(H)+\sum_{t=1}^{T-2} Y_{t}+\mathbb{E}_{T-2}^{*}\left(\frac{1-a_{T-1}^{*} X_{T-1}}{1-b_{T-1}^{*}} Y_{T-1}\right) \\
& +\mathbb{E}_{T-2}^{*}\left(\frac{1-a_{T-1}^{*} X_{T-1}}{1-b_{T-1}^{*}} \frac{1-a_{T}^{*} X_{T}}{1-b_{T}^{*}} Y_{T}\right) .
\end{aligned}
$$

Iterating the process yields the required result for $V_{0}^{*}$.

Now, we assume the existence of a traded insurance derivative which we identify with the first traded risky asset. The derivative is written at each time $t-1$ and pays 1 unit at time $t$, if the claims development result $Y_{t}$ exceeds a given high threshold $d_{t}$. Specifically,

$$
X_{t}^{(1)}=\frac{\mathbf{1}_{D_{t}}}{q_{t}}-1,
$$

where $D_{t}=\left\{Y_{t} \geq d_{t}\right\}, \mathbb{E}_{t-1}\left(\mathbf{1}_{D_{t}}\right)=p_{t}$, and $q_{t}$ is the $\mathcal{F}_{t-1}$-measurable price at time $t-1$ with $p_{t}<q_{t}<1$. In fact, much of the following analysis remains unchanged if we assume, similarly to Section 2 , that the event $D_{t}=\left\{Z_{t} \geq d_{t}\right\}$, where $Z_{t}$ is an (index) variable closely correlated to $Y_{t}$. For the sake of simplicity, we do not pursue this route here.

Additional assumptions give rise to formulas generalizing those of Section 2. 
Proposition 4. Let $X_{t}^{(1)}$ be as in (16). Assume that $a_{t}$ and $b_{t}$ are state independent and that for any $1 \leq i, j \leq n$ and $0<t<t+s \leq T$, it is

$$
\mathbb{E}_{t-1}\left(X_{t}^{(i)} X_{t+s}^{(j)} Y_{t+s}\right)=\mathbb{E}_{t-1}\left(X_{t}^{(i)}\right) \mathbb{E}_{t-1}\left(X_{t+s}^{(j)} Y_{t+s}\right), \quad \mathbb{E}_{t-1}\left(X_{t}^{(i)} Y_{t+s}\right)=0
$$

Then, the optimal initial endowment of Theorem 2 becomes

$$
\begin{aligned}
V_{0} & =\mathbb{E}_{0}(H)+\sum_{t=1}^{T} \mathbb{E}_{0}\left(\frac{1-a_{t} X_{t}}{1-b_{t}} Y_{t}\right) \\
& =\mathbb{E}_{0}(H)-\sum_{t=1}^{T} \frac{a_{t}^{(1)}}{1-b_{t}} \mathbb{E}_{0}\left(\frac{p_{t}}{q_{t}} \operatorname{TVaR}_{1-p_{t}, t-1}\left(Y_{t}\right)\right)-\sum_{t=1}^{T} \sum_{i=2}^{n} \frac{a_{t}^{(i)} \operatorname{Cov}_{0}\left(X_{t}^{(i)}, Y_{t}\right)}{1-b_{t}},
\end{aligned}
$$

where $\mathrm{TVaR}_{1-p_{t}, t-1}$ is the risk measure calculated with respect to information $\mathcal{F}_{t-1}$ at time $t-1$.

Proof. The uncorrelatedness assumption and normalization imply

$$
\mathbb{E}_{T-2}\left(\frac{1-a_{T-1} X_{T-1}}{1-b_{T-1}} \frac{1-a_{T} X_{T}}{1-b_{T}} Y_{T}\right)=\mathbb{E}_{T-2}\left(\frac{1-a_{T} X_{T}}{1-b_{T}} Y_{T}\right) .
$$

The proof of the first statement then follows from Proposition 3 working backward in time. The second formula derives from

$$
\begin{aligned}
\mathbb{E}_{0}\left(X_{t}^{(1)} Y_{t}\right) & =\mathbb{E}_{0}\left(\frac{1}{q_{t}} \mathbf{1}_{D_{t}} Y_{t}\right)=\mathbb{E}_{0}\left(\frac{p_{t}}{q_{t}} \frac{1}{p_{t}} \mathbb{E}_{t-1}\left(\mathbf{1}_{D_{t}} Y_{t}\right)\right) \\
& =\mathbb{E}_{0}\left(\frac{p_{t}}{q_{t}} \operatorname{TVaR}_{1-p_{t}, t-1}\left(Y_{t}\right)\right) .
\end{aligned}
$$

The conditions of Proposition 4 correspond, loosely speaking, to the assumption that the conditional expected performance of assets over each time period is already known at time $t=0$ and that assets and liabilities are uncorrelated across time periods. Then, the market value $V_{0}$ of the liability $H$ equals its expected value plus a number of terms producing valuations of the individual claims development results $Y_{t}$. Each of the latter terms can be written as a weighted sum of the expected value of a TVaR measure applied to $Y_{t}$ at time $t-1$, scaled by $p_{t} / q_{t}$, and $n-1$ CAPM-type terms corresponding to the other tradeable assets. Thus, the valuation formula of Proposition 4 bears a formal similarity to commonly used multi-period cost-of-capital formulas termed as the split of total uncertainty approach in Salzmann and Wüthrich [20] or expected risk margin in Möhr [16]. At the same time, it generalizes them by including further tradeable assets via standard valuation arguments.

If we do not consider any tradeable assets except the derivatives on $Y_{t}$ ( $n=$ 1), a further simplification arises. It is easily shown that $a_{t}=\frac{p_{t}-q_{t}}{q_{t}^{2}+p_{t}-2 q_{t} p_{t}}$ and 
$b_{t}=\frac{\left(p_{t}-q_{t}\right)^{2}}{q_{t}^{2}+p_{t}-2 q_{t} p_{t}}$. Moreover, if $a_{t}, b_{t}$ are state independent, so are $p_{t}, q_{t}$, such that a single-asset and multi-period valuation formula, directly generalizing (8), is obtained:

$$
V_{0}=\mathbb{E}_{0}(H)+\sum_{t=1}^{T} \frac{q_{t}-p_{t}}{1-p_{t}} \operatorname{TVaR}_{1-p_{t}}\left(Y_{t}\right) .
$$

A comment is relating due to the uncorrelatedness assumption of Proposition 4. For simplicity, consider the single-asset case. Then, the proposition requires $\mathbb{E}_{t-2}\left(\mathbf{1}_{\left\{Y_{t-1} \geq d_{t-1}\right\}} Y_{t}\right)=0$ and $\mathbb{E}_{t-2}\left(\mathbf{1}_{\left\{Y_{t-1} \geq d_{t-1}\right\}} \mathbf{1}_{\left\{Y_{t} \geq d_{t}\right\}} Y_{t}\right)=$ $\mathbb{E}_{t-2}\left(\mathbf{1}_{\left\{Y_{t-1} \geq d_{t-1}\right\}}\right) \mathbb{E}_{t-2}\left(\mathbf{1}_{\left\{Y_{t} \geq d_{t}\right\}} Y_{t}\right)$. While the random variables $Y_{t-1}$ and $Y_{t}$ are uncorrelated (due to the martingale property), this does not necessarily imply that the pairs of random variables $\left(\mathbf{1}_{\left\{Y_{t-1} \geq d_{t-1}\right\}}, Y_{t}\right)$ and $\left(\mathbf{1}_{\left\{Y_{t-1} \geq d_{t-1}\right\}}, \mathbf{1}_{\left\{Y_{t} \geq d_{t}\right\}} Y_{t}\right)$ are also uncorrelated. Stronger assumptions on the joint distribution of the vector $\left(Y_{1}, \ldots, Y_{T}\right)$ are thus required, for instance it is sufficient to assume that the martingale innovations are independent.

Two numerical examples are now presented. In Example 3, a direct application of Proposition 4 is given for the case of two assets and several time periods. In Example 4, we discuss the case where $a_{t}, b_{t}$ are not state independent. In particular, we assume a scenario where, though the random variables $Y_{t}$ are independent of each other, markets take a different view such that a high level of $Y_{t-1}$ is associated with a high market price $q_{t}$ for the pay-off $\mathbf{1}_{\left\{Y_{t} \geq d_{t}\right\}}$.

Example 3. In this example, we consider a long-term $\mathcal{F}_{T}$-measurable liability $H$ with $\mathbb{E}_{0}(H)=100, T=10$ years and two tradeable assets in each period. These are a derivative with price at time $t-1$ of $q_{t}$ and pay-off at time $t$ of $\mathbf{1}_{\left\{Y_{t} \geq d_{t}\right\}}$ and a stock with price process $S_{t}^{(2)}$ and excess return $X_{t}^{(2)}$. We assume that the claims development results $Y_{1}, \ldots, Y_{T}$ are mutually independent and so are their derivative returns $X_{1}^{(1)}, \ldots, X_{T}^{(1)}$. Moreover, the pair $\left(Y_{t}, X_{t}^{(2)}\right)$ is defined via a bivariate log-normal model, such that

$$
Y_{t}=\exp \left(\mu_{t}+\sigma_{t} Z_{t}^{(1)}\right)-\exp \left(\mu_{t}+\sigma_{t}^{2} / 2\right) \quad \text { and } \quad X_{t}^{(2)}=\exp \left(m+s Z_{t}^{(2)}\right)-1,
$$

where $\left(Z_{t}^{(1)}, Z_{t}^{(2)}\right)$ follow a bivariate standard normal distribution with correlation $r$. This implies that we can write $Z_{t}^{(2)}=r Z_{t}^{(1)}+\sqrt{1-r^{2}} W_{t}$, where $\left(Z_{t}^{(1)}, W_{t}\right)$ are independent standard normal variables. Note that, as required, $\mathbb{E}_{t-1}\left(Y_{t}\right)=0$. The model for $Y_{t}$ used here is illustrative, as in a more realistic application one would need to derive the dynamics of $Y_{t}$ from a stochastic reserving model, see for instance Merz et al. [15].

For the derivative we use parameters $p_{t}=0.01$ and $q_{t}=0.066$ for all $t$, implying that the threshold $d_{t}$ is always set at the 99th percentile of $Y_{t}$ and that the derivative price in future periods is assumed constant. For the claims development results, we use $\mu_{t}=0.4586(T-t+1)$ and $\sigma_{t}=0.198$ for all $t$, such that the standard deviation of $Y_{t}$ reduces over time, reflecting that uncertainty 


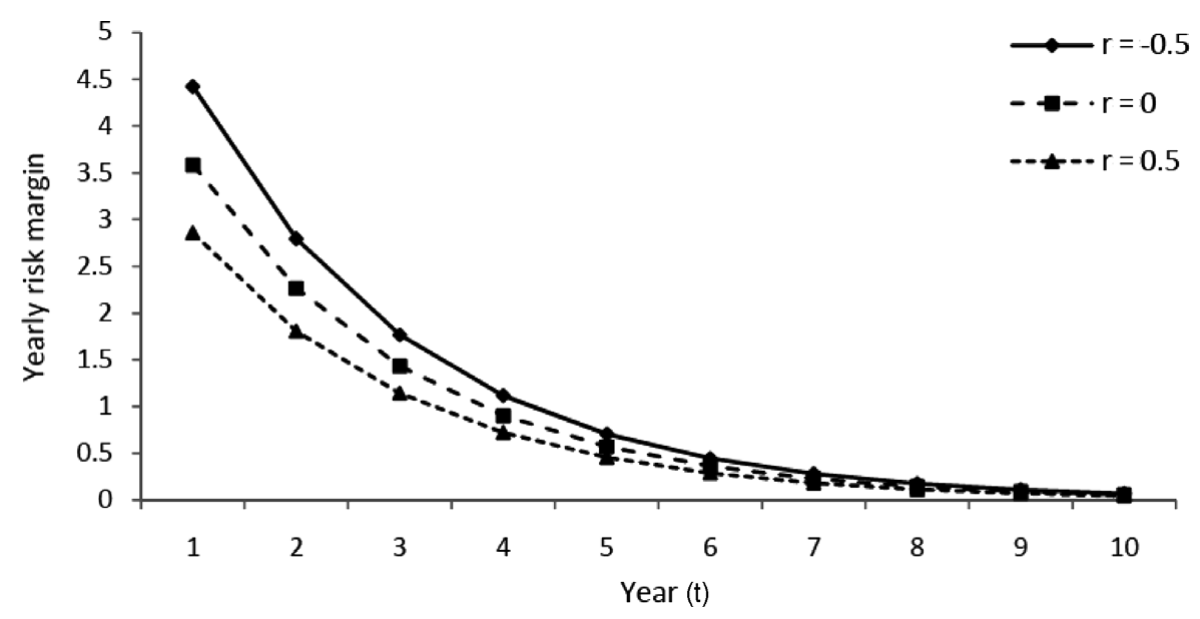

FIGURE 2: Yearly risk margins for different levels of correlation parameter $r$.

decreases with increasing information. For the stock, we use $m=0.15$ and $s=0.2$. Finally, $r$ is allowed to vary in the range $(-1,1)$. A positive (negative) correlation corresponds to the situation when stock prices tend to increase (decrease) at times of high claims development results (motivated by economically driven claims inflation).

We proceed by applying Proposition 4 . The necessary calculations are somewhat tedious and are documented in the Appendix. For the correlation parameter values $r \in\{-0.5,0,0.5\}$, market values of $H$ equaling $V_{0}(r)=$ $\{111.90,109.64,107.69\}$, respectively, are obtained. In Figure 2, we plot the market risk margin applied for each year of the liability's run-off, that is, the quantities $\sum_{i=1}^{2} \frac{a_{t}^{(i)} \operatorname{Cov}_{0}\left(X_{t}^{(i)}, Y_{t}\right)}{1-b_{t}}, t=1, \ldots, T$.

We observe that the case $r=0$ is equivalent to the absence of the stock such that $V_{0}$ is given by expression (17). This means that no risk in the claims development result can be mitigated by the asset stock price process. When $r=$ 0.5 , long positions in the stock produce a natural hedging effect for the liability as investment returns pay for claims development results. This situation, which is desirable for the holder of the liability, decreases $V_{0}$ in relation to the case $r=0$. Conversely, when $r=-0.5$, short positions in the stock are taken. Thus, in order to hedge the liability, negative expected stock returns are incurred. This adverse situation, analogous to the liability being subject to systematic risk, increases $V_{0}$ in relation to the case $r=0$. It can be seen from Figure 2 that the annual contributions to the market value of $H$ decrease with time. This is explained by the decay of the standard deviation of $Y_{t}$ in our model as $t$ increases.

Example 4. To avoid computational issues, we now consider a shorter term liability $H$, with $T=2$ and $\mathbb{E}_{0}(H)=100$. In this example, there is no stock correlated with claims development results such that the only tradeable asset is the derivative on $Y_{1}$ and $Y_{2}$. Again, we assume that the claims development 
results are mutually independent and

$$
Y_{t}=\exp \left(\mu_{t}+\sigma_{t} Z_{t}\right)-\exp \left(\mu_{t}+\sigma_{t}^{2} / 2\right), \quad t=1,2,
$$

where $Z_{1}, Z_{2}$ are independent standard normals. The parameters of the claims development results are $\mu_{1}=4.586, \mu_{2}=4.127, \sigma_{1}=\sigma_{2}=0.198$.

We now consider a derivative with a higher probability of a pay-off than in the previous example such that $p_{1}=p_{2}=0.05$ and $q_{1}=0.21$. However, $q_{2}$ is no longer known at time $t=0$, but is instead dependent on $Y_{1}$. If the derivative produces a pay-off, the market price of the derivative increases in the next period (and vice versa). Specifically, we define $q_{2}$ by

$$
q_{2}=\left\{\begin{array}{l}
\underline{q} \leq q_{1}, \text { if } Y_{1}<d_{1}, \\
\bar{q} \geq q_{1}, \text { if } Y_{1} \geq d_{1} .
\end{array}\right.
$$

To aid comparisons, we let $\mathbb{E}_{0}\left(q_{2}\right)=\left(1-p_{1}\right) q+p_{1} \bar{q}=q_{1}$. The sensitivity of $q_{2}$ on past performance of the derivative is studied by considering three cases: (i) $\bar{q} / q=1$ giving $q=\bar{q}=0.21$; (ii) $\bar{q} / q=2$ giving $q=0.2, \bar{q}=0.4$; and (iii) $\bar{q} / \underline{q}=4$ giving $\underline{q}=0.183, \bar{q}=0.730$.

To calculate the market value $V_{0}^{*}$, we use Theorem 2. In particular, we have $L_{2}=1$ and

$$
\begin{aligned}
a_{2}^{*} & =a_{2}=\frac{\mathbb{E}_{1}\left(X_{2}\right)}{\mathbb{E}_{1}\left(X_{2}^{2}\right)}=\frac{p_{2}-q_{2}}{p_{2}+q_{2}^{2}-2 p_{2} q_{2}}, \\
b_{2}^{*} & =b_{2}=\frac{\left(\mathbb{E}_{1}\left(X_{2}\right)\right)^{2}}{\mathbb{E}_{1}\left(X_{2}^{2}\right)}=\frac{\left(p_{2}-q_{2}\right)^{2}}{p_{2}+q_{2}^{2}-2 p_{2} q_{2}}, \\
V_{1}^{*} & =V_{1}=\mathbb{E}_{1}\left(\frac{1-a_{2} X_{2}}{1-b_{2}} H\right)=\mathbb{E}_{0}(H)+Y_{1}+\mathbb{E}_{1}\left(\frac{1-a_{2} X_{2}}{1-b_{2}} Y_{2}\right) \\
& =\mathbb{E}_{0}(H)+Y_{1}+\frac{q_{2}-p_{2}}{1-p_{2}} \operatorname{TVaR}_{1-p_{2}}\left(Y_{2}\right) .
\end{aligned}
$$

Hence, $V_{1}^{*}$ can be explicitly calculated as a function of $q_{2}$, which is in turn a function of $Y_{1}$. To derive $V_{0}^{*}$, we need to calculate $L_{1}=1-b_{2}$ and, observe that $d \mathbb{P}_{0}^{*}=L_{1} / \mathbb{E}_{0}\left[L_{1}\right] d \mathbb{P}_{0}$,

$$
\begin{aligned}
a_{1}^{*} & =\frac{\mathbb{E}_{0}\left(L_{1} X_{1}\right)}{\mathbb{E}_{0}\left(L_{1} X_{1}^{2}\right)} \quad \text { and } \quad b_{1}^{*}=\frac{\mathbb{E}_{0}\left(L_{1} X_{1}\right)^{2}}{\mathbb{E}_{0}\left(L_{1} X_{1}^{2}\right) \mathbb{E}_{0}\left(L_{1}\right)}, \\
V_{0}^{*} & =\frac{1}{\mathbb{E}_{0}\left(L_{1}\right)} \mathbb{E}_{0}\left(L_{1} \frac{1-a_{1}^{*} X_{1}}{1-b_{1}^{*}} V_{1}^{*}\right) .
\end{aligned}
$$

These calculations of the market value $V_{0}^{*}$ can be easily done by Monte Carlo simulation. Using a simulated sample of $5 \cdot 10^{6}$ from $Y_{1}$, we obtain that (i) for 
$\bar{q} / q=1$, it is $V_{0}^{*}=113.2$; (ii) for $\bar{q} / q=2$, it is $V_{0}^{*}=114.2$; and (iii) for $\bar{q} / q=4$, it is $V_{0}^{*}=116.0$.

Hence, with increasing sensitivity of $q_{2}$ to the outcome of $Y_{1}$, the market value of the liability increases. Intuitively, this is clear that the uncertainty is increased by increasing price sensitivity in $q_{2}$ in terms of $Y_{1}$. This case may be more realistic in comparison to a scenario where derivative prices are unaffected by observed losses, that is, where $q_{2}$ does not depend on the outcome of $Y_{1}$, because investors react sensitively based on past observations. However, at least for this short tail example, the increase is not particularly dramatic.

\subsection{Hedging and capital efficiency}

In Section 2.3, the issue of capital efficiency was discussed in relation to the single-period model. The relation between hedging and capital efficiency becomes rather convoluted in the multi-period case. The reason for this is structural. While capital requirements in insurance are typically calculated with respect to a one-year time horizon, the optimal investment strategy is formulated to minimize a quadratic error calculated at the time horizon $T$. In particular, the trading strategy in each period will also reflect the performance of the portfolio to-date, which introduces path-dependency.

Consider the simplest possible case, where $Y_{1}, \ldots, Y_{T}$ are independent, $a_{t}, b_{t}$ are $\mathcal{F}_{0}$-measurable, and the only traded asset is the derivative on $Y_{t}$. Then, from Theorem 2, it is seen that the optimal trading strategy for initial endowment $V_{0}$ is given by

$$
\phi_{t}\left(V_{0}\right)=\xi_{t}+a_{t}\left(V_{t-1}-G_{t-1}^{V_{0}, \phi\left(V_{0}\right)}\right), \quad \text { where } \xi_{t}=\frac{\mathbb{E}_{t-1}\left(\left(V_{t}-V_{t-1}\right) X_{t}\right)}{\mathbb{E}_{t-1}\left(X_{t}^{2}\right)} .
$$

Straightforward but tedious calculations then yield $\xi_{t}=\frac{\operatorname{Cov}_{0}\left(X_{t}, Y_{t}\right)}{\operatorname{Var}_{0}\left(X_{t}\right)}$. Hence, the trading strategy $\phi_{t}\left(V_{0}\right)$ consists of two parts: $\xi_{t}$, the values of which in this simple setting are known at time 0 , and $a_{t}\left(V_{t-1}-G_{t-1}^{V_{0}, \phi\left(V_{0}\right)}\right)$, which reflects the value of the investment portfolio at time $t$. Note that $\xi_{t}$ is essentially identical to $\xi_{1}$ in (2). Let $\delta_{t}=V_{t-1}-G_{t-1}^{V_{0}, \phi\left(V_{0}\right)}$ represent the difference between the value of the liability and the value of the investment portfolio at time $t-1$. Then, since $\mathbb{E}_{t-1}\left(X_{t}\right) \leq 0 \Longrightarrow a_{t} \leq 0$, in the multi-period case we adjust the trading strategy such that, if the shortfall is $\delta_{t}>0$, less is invested in the risky asset and vice versa.

Analogously to what was discussed in Section 2.3, a plausible re-formulation of the capital efficiency condition (10) at time $t-1$ is

$$
\rho_{t-1}\left(Y_{t}-\left(G_{t}^{V_{0}, \phi\left(V_{0}\right)}-G_{t-1}^{V_{0}, \phi\left(V_{0}\right)}\right)\right) \leq \rho_{t-1}\left(Y_{t}\right),
$$

where $\rho_{t-1}$ is the regulatory risk measure evaluated given the information $\mathcal{F}_{t-1}$ available at time $t-1$. The principle here is that the liability with respect to which capital needs to be held during $(t-1, t]$ is the corresponding claims development 
result $Y_{t}$. Inequality (18) represents the condition that the capital required to support $Y_{t}$, minus the net gains from trading over the same interval, is less than the capital required to support $Y_{t}$, assuming that all funds are invested in the risk-free asset. The left-hand side of inequality (18) can be written as

$$
\rho_{t-1}\left(Y_{t}-\phi_{t}\left(V_{0}\right) X_{t}\right)=\rho_{t-1}\left(Y_{t}-\xi_{t} X_{t}-a_{t} \delta_{t} X_{t}\right) .
$$

Define $\tilde{k}_{t}=a_{t} \delta_{t}+\frac{1}{1-p_{t}} \operatorname{TVaR}_{t-1,1-p_{t}}\left(Y_{t}\right)$. Then, retracing the first steps in the proof of Proposition 1, it follows that the condition for inequality (18) to hold is, analogously to (11),

$$
q_{t} \tilde{k}_{t} \leq \rho_{t-1}\left(Y_{t}\right)-\rho_{t-1}\left(Y_{t}-\tilde{k}_{t} \mathbf{1}_{D_{t}}\right) .
$$

Finally, we remark that $\xi_{t}$ corresponds exactly to the investment in the stock under the (non-self-financing) local risk-minimizing hedging strategy of Föllmer and Schweizer [11]. Therefore, under such a trading strategy with explicit one-period optimization targets, the present discussion of capital efficiency would be much simplified.

\section{CONCLUDING REMARKS}

We discussed the problem of valuing insurance liabilities in discrete time through mean-variance hedging. Key features of the proposed approach are the decomposition of the terminal liability into claims development results and the presence of a derivative on the claims development result in each period. In simple cases, the resulting valuation formulas become structurally very similar to regulatory cost-of-capital-based formulas. However, adoption of the meanvariance framework improves upon such formulas, by introducing sensitivity to observed market prices, the inclusion of other tradeable assets, and the consistent extension to multiple periods.

The similarity between the formulas derived here and the ones used in regulation should not obscure the very different interpretations underlying them. In our approach, the market value margin obtained (difference between market consistent and expected values) does not correspond to the cost-of-capital, but reflects the cost of a replication portfolio. Hence, it is conceivable that a costof-capital loading may be added to the market consistent value that we obtain, since investors need to be compensated for the frictional costs that holding capital incurs (see e.g. the discussions in Zanjani [27] and Venter [25]). The analysis of Section 2.3 shows that the mean-variance hedging approach may also deliver a reduction in such capital costs.

It is then useful to distinguish between the possible constituent parts of the value of a liability. Thus, if a cost-of-capital loading is added to the (partial) replication cost that our valuation formulas reflect, this should only represent frictional capital costs. In particular, it should not be further increased to act as a proxy for replication costs, as current regulatory valuation approaches implicitly 
do. Finally, besides the cost of replication and the frictional cost-of-capital, it is plausible that an additional risk load is applied via a performance measure, purely to reward investors for risk taking. This need not be related to a tail risk measure like VaR or TVaR; for example, mean-variance hedging approaches can be adjusted to deliver a pre-specified minimal level of Sharpe ratio (Černý [1], Section 13.2).

\section{ACKNOWLEDGEMENTS}

The authors would like to thank a referee for insightful observations that improved the paper.

\section{REFERENCES}

[1] ČERnÝ, A. (2009) Mathematical Techniques in Finance. Princeton: Princeton University Press.

[2] ČERNÝ, A. and KALLSEN, J. (2007) On the structure of general mean-variance hedging strategies. Annals of Probability, 35(4), 1479-1531.

[3] ČERnÝ, A. and KALLSEN, J. (2009) Hedging by sequential regression revisited. Mathematical Finance, 19(4), 591-617.

[4] Cummins, J.D. (2008) CAT bonds and other risk-linked securities: State of the market and recent developments. Risk Management and Insurance Review, 11(1), 23-47.

[5] DAHL, M., MøLLER, T. (2006) Valuation and hedging of life insurance liabilities with systematic mortality risk. Insurance: Mathematics and Economics, 39(2), 193-217.

[6] Denuit, M., Dhaene, J., Goovaerts, M. and KaAs, R. (2005) Actuarial Theory for Dependent Risks: Measures, Orders and Models. England: Wiley.

[7] Delong, Ł. (2012) No-good-deal, local mean-variance and ambiguity risk pricing and hedging for an insurance payment process. ASTIN Bulletin, 42(1), 203-232.

[8] Delong, E. and Gerrard, R. (2007) Mean-variance portfolio selection for a non-life insurance company. Mathematical Methods of Operations Research, 66, 339-367.

[9] DOHERTY, N.A. (1997) Innovations in managing catastrophe risk. Journal of Risk and Insurance, 64(4), 713-718.

[10] European Commission (2010) QIS 5 Technical Specifications, Annex to Call for Advice from CEIOPS on QIS5.

[11] FÖllmer H. and SCHWEIZER, M. (1988) Hedging by sequential regression: An introduction to the mathematics of option trading. ASTIN Bulletin, 18(2), 147-160.

[12] Haslip, G.G. and KAishev, V. K. (2010) Pricing of reinsurance contracts in the presence of catastrophe bonds. ASTIN Bulletin, 40(1), 307-329.

[13] McNeil, A.J., Frey, R. and Embrechts, P. (2005) Quantitative Risk Management: Concepts, Techniques and Tools. Princeton: Princeton University Press.

[14] MERZ, M. and WÜTHRICH, M.V. (2008) Modelling the claims development result for solvency purposes. Casualty Actuarial Society E-Forum, Fall 2008, 542-568.

[15] Merz, M., WÜthrich, M.V. and Hashorva, E. (2013) Dependence modeling in multivariate claims run-off triangles. Annals of Actuarial Science, 7(1), 3-25.

[16] MöHR, C. (2011) Market-consistent valuation of insurance liabilities by cost of capital. ASTIN Bulletin, 41(2), 315-341.

[17] MøLleR, T. (1998) Risk-minimizing hedging strategies for unit-linked life insurance contracts. ASTIN Bulletin, 28(1), 17-47.

[18] MøLlER, T. (2001) Risk-minimizing hedging strategies for insurance payment processes. Finance and Stochastics, 5(4), 419-446. 
[19] PAPAChristou, D. (2011) Statistical analysis of the spreads of catastrophe bonds at the time of issue. ASTIN Bulletin, 41(1), 251-277.

[20] SAlzmanN, R. and WÜTHRICH, M.V. (2010) Cost-of-capital margin for a general insurance liability runoff. ASTIN Bulletin, 40(2), 415-451.

[21] SchweIzer, M. (2001) A guided tour through quadratic hedging approaches. In Option Pricing, Interest Rates and Risk Management (eds. E. Jouini, J. Cvitanić and M. Musiela), pp. 538-574. Cambridge, UK: Cambridge University Press.

[22] SChweizer, M. (2001) From actuarial to financial valuation principles. Insurance: Mathematics and Economics, 28(1), 31-47.

[23] SwISS SOlVENCY TeST (2006) FINMA SST Technisches Dokument, Version 2. October 2006.

[24] THOMSON, R.J. (2005) The pricing of liabilities in an incomplete market using dynamic meanvariance hedging. Insurance: Mathematics and Economics, 36(3), 441-455.

[25] Venter, G.G. (2004) Capital allocation survey with commentary. North American Actuarial Journal, 8(2), 96-107.

[26] Wüthrich, M.V., Merz, M. (2013) Financial Modeling, Actuarial Valuation and Solvency in Insurance. Berlin: Springer.

[27] ZANJANI, G. (2002) Pricing and capital allocation in catastrophe insurance. Journal of Financial Economics, 65, 283-305.

ANDREAS TSANAKAS (Corresponding author)

Cass Business School, City University London, 106 Bunhill Row,

London EC1 Y 8TZ, UK

E-Mail: a.tsanakas.1@city.ac.uk

MARIO V. WÜTHRICH

Department of Mathematics, ETH Zurich, RiskLab, 8092 Zurich, Switzerland

ALEŠ ČERNÝ

Cass Business School, City University London, 106 Bunhill Row, London EC1 Y 8TZ, UK

\section{APPENDIX}

\section{CALCULATIONS IN EXAMPLE 3}

For the calculations shown here, it is convenient to use excess returns $X_{t}$ rather than price increments, as discussed in Section 3.1. To determine $V_{0}$, we first need to calculate all terms in

$$
a_{t}=\mathbb{E}_{t-1}\left(X_{t}^{\prime}\right) \mathbb{E}_{t-1}\left(X_{t} X_{t}^{\prime}\right)^{-1} \quad \text { and } \quad b_{t}=\mathbb{E}_{t-1}\left(X_{t}^{\prime}\right) \mathbb{E}_{t-1}\left(X_{t} X_{t}^{\prime}\right)^{-1} \mathbb{E}_{t-1}\left(X_{t}\right)
$$

Model assumption $Y_{t}=\exp \left(\mu_{t}+\sigma_{t} Z_{t}^{(1)}\right)-\exp \left(\mu_{t}+\sigma_{t}^{2} / 2\right)$ provides returns

$$
X_{t}^{(1)}=\frac{1}{q_{t}} \mathbf{1}_{\left\{Y_{t} \geq d_{t}\right\}}-1, \quad X_{t}^{(2)}=\exp \left(m+s Z_{t}^{(2)}\right)-1,
$$


where $Z_{t}^{(2)}=r Z_{t}^{(1)}+\sqrt{1-r^{2}} W_{t}$ and $\left(Z_{t}^{(1)}, W_{t}\right)$ are independent standard normals. Therefore, to apply Proposition 4 we need to calculate the first and second moments of $X_{t}$ as well as the covariances $\operatorname{Cov}_{t-1}\left(X_{t}^{(i)}, Y_{t}\right)$ for $i=1,2$. The first moments of $X_{t}$ are given by

$$
\mathbb{E}_{t-1}\left(X_{t}^{(1)}\right)=\frac{p_{t}}{q_{t}}-1 \quad \text { and } \quad \mathbb{E}_{t-1}\left(X_{t}^{(2)}\right)=\exp \left(m+s^{2} / 2\right)-1
$$

The second moments of $X_{t}$ are given by

$$
\begin{aligned}
\mathbb{E}_{t-1}\left(\left(X_{t}^{(1)}\right)^{2}\right) & =\frac{1}{q_{t}^{2}} p_{t}\left(1-p_{t}\right)+\left(\frac{p_{t}}{q_{t}}-1\right)^{2}, \\
\mathbb{E}_{t-1}\left(\left(X_{t}^{(2)}\right)^{2}\right) & =1-2 \exp \left(m+s^{2} / 2\right)+\exp \left(2 m+2 s^{2}\right), \\
\mathbb{E}_{t-1}\left(X_{t}^{(1)} X_{t}^{(2)}\right) & =1-\exp \left(m+s^{2} / 2\right)-\frac{p_{t}}{q_{t}}+\mathbb{E}_{t-1}\left(\frac{1}{q_{t}} \mathbf{1}_{\left\{Y_{t} \geq d_{t}\right\}} \exp \left(m+s Z_{t}^{(2)}\right)\right) .
\end{aligned}
$$

Let $\tilde{d}_{t}=d_{t}+\exp \left(\mu_{t}+\sigma_{t}^{2} / 2\right)=\exp \left(\mu_{t}+\sigma_{t} \Phi^{-1}\left(1-p_{t}\right)\right)$, where $\Phi$ is the standard normal distribution. Then $\mathbf{1}_{\left\{Y_{t} \geq d_{t}\right\}}=\mathbf{1}_{\left\{\exp \left(\mu_{t}+\sigma_{t} Z_{t}^{(1)}\right) \geq \tilde{d}_{t}\right\}}$, such that

$$
\mathbb{E}_{t-1}\left(\frac{1}{q_{t}} \mathbf{1}_{\left\{Y_{t} Y \geq d_{t}\right\}} \exp \left(m+s Z_{t}^{(2)}\right)\right)=\frac{1}{q_{t}}\left(\exp \left(m+s^{2}\left(1-r^{2}\right)\right) \cdot g(r),\right.
$$

where we have defined $g(r)=\mathbb{E}_{t-1}\left(\mathbf{1}_{\left\{Z_{t}^{(1)} \geq\left(\log \tilde{d}_{t}-\mu_{t}\right) / \sigma_{t}\right\}} \exp \left(\operatorname{sr} Z_{t}^{(1)}\right)\right)$. From the definition of $\tilde{d}_{t}$, we obtain for $r=0$ the value $g(0)=p_{t}$. Denote $k=\left(\log \tilde{d}_{t}-\mu_{t}\right) / \sigma_{t}$. If $r>0$, we have $g(r)=\exp \left(s^{2} r^{2} / 2\right) \Phi\left(\frac{s^{2} r^{2}-s r k}{s r}\right)$, using the properties of the log-normal distribution. If $r<0$, we have $g(r)=\exp \left(s^{2} r^{2} / 2\right)\left[1-\Phi\left(\frac{s^{2} r^{2}-s r k}{-s r}\right)\right]$.

Finally, we move to the calculation of the covariances. They are given by

$$
\begin{aligned}
\operatorname{Cov}_{t-1}\left(X_{t}^{(1)}, Y_{t}\right) & =\mathbb{E}_{t-1}\left(\frac{1}{q_{t}} \mathbf{1}_{\left\{\exp \left(\mu_{t}+\sigma_{t} Z_{t}^{(1)}\right) \geq \tilde{d}_{t}\right\}} \exp \left(\mu_{t}+\sigma_{t} Z_{t}^{(1)}\right)\right)-\frac{p_{t}}{q_{t}} \exp \left(\mu_{t}+\sigma_{t}^{2} / 2\right) \\
& =\frac{1}{q_{t}} \exp \left(\mu_{t}+\sigma_{t}^{2} / 2\right) \Phi\left(\frac{\mu_{t}+\sigma_{t}^{2}-\log \tilde{d}_{t}}{\sigma_{t}}\right)-\frac{p_{t}}{q_{t}} \exp \left(\mu_{t}+\sigma_{t}^{2} / 2\right),
\end{aligned}
$$

and

$$
\begin{aligned}
\operatorname{Cov}_{t-1}\left(X_{t}^{(2)}, Y_{t}\right)= & \mathbb{E}_{t-1}\left(\exp \left(m+\mu_{t}+\left(s r+\sigma_{t}\right) Z_{t}^{(1)}+s \sqrt{1-r^{2}} W_{t}\right)\right) \\
& -\exp \left(m+\mu_{t}+\left(s^{2}+\sigma_{t}^{2}\right) / 2\right) \\
= & \exp \left(m+\mu_{t}+\left(s r+\sigma_{t}\right)^{2} / 2+s^{2}\left(1-r^{2}\right) / 2\right) \\
& -\exp \left(m+\mu_{t}+\left(s^{2}+\sigma_{t}^{2}\right) / 2\right) .
\end{aligned}
$$

This completes the required calculations. 